\title{
Gamma-rays and neutrinos from TXS 0506+056: interpretation of the 2018/2019 MAGIC and MWL monitoring campaign
}

\author{
E. Bernardini ${ }^{a}$, W. Bhattacharyya ${ }^{b}$, M. Cerruti ${ }^{*}$, , V. Fallah Ramazani ${ }^{d}$, L. Foffano $^{a}, \mathbf{S}$. \\ Inoue $^{e}$, E. Prandini ${ }^{a}$, Ch. Righi ${ }^{f}$, K. Satalecka ${ }^{b}$, N. Sahakyan ${ }^{g}$, F. Tavecchio ${ }^{f}$, for the \\ MAGIC Collaboration ${ }^{\dagger}$ \\ ${ }^{a}$ Università di Padova and INFN, I-35131 Padova, Italy; \\ ${ }^{b}$ DESY, 15738 Zeuthen, Germany; \\ ${ }^{c}$ Universitat de Barcelona, ICCUB, IEEC-UB, E-08028 Barcelona, Spain ${ }^{d}$ Finnish Centre of \\ Astronomy with ESO (FINCA), University of Turku, FI-20014 Turku, Finland; \\ e ICRR, The University of Tokyo, 277-8582 Chiba, Japan; \\ ${ }^{f}$ National Institute for Astrophysics (INAF), I-00136 Rome, Italy; \\ ${ }^{g}$ ICRANet-Armenia at NAS RA, 0019 Yerevan, Armenia \\ E-mail: matteo.cerruti@icc.ub.edu
}

\begin{abstract}
A powerful tool to investigate the sources of cosmic-rays is multi-messenger astronomy. If cosmic rays are accelerated in relativistic jets of active galactic nuclei, they interact with low-energy photon fields producing photons and neutrinos that can be used to infer the properties of the hadronic accelerator. On 09/22/2017, IceCube and the gamma-ray instruments Fermi-LAT and MAGIC observed the first evidence (at the 3 sigma level) of co-production of photons and neutrinos from the AGN TXS 0506+056. For the first time, theoretical models of hadronic emission from AGNs could be tested on both gamma-rays and neutrinos. Following this event, the MAGIC collaboration set up a gamma-ray monitoring of the source during the 2018-2019 observing season, accompanied by multi-wavelength observations, resulting in a new MAGIC detection and producing new quasi-simultaneous spectral energy distributions of the source. In this contribution we present the theoretical interpretation of the 2018-2019 observations of TXS 0506+056, comparing the results from this new data-set with the one from September 2017, and we discuss their implications on cosmic-ray accelerations in $\mathrm{AGN}$, and on the physics of relativistic jets from super-massive black holes.
\end{abstract}

36th International Cosmic Ray Conference -ICRC2019-

July 24th - August 1st, 2019

Madison, WI, U.S.A.

\footnotetext{
* Speaker.

†https://magic.mpp.mpg.de/. For collaboration list see PoS(ICRC2019)1177
} 